\title{
AT1 receptors modulate AKI
}

The renin-angiotensin system (RAS) has been implicated in the pathogenesis of acute kidney injury (AKI), but conflicting outcomes have been reported in patients following RAS inhibition. New data from Steven Crowley and colleagues suggest that type 1 angiotensin II (AT1) receptors have differential effects on T cells and kidney epithelial cells during cisplatin-induced $\mathrm{AKI}$, and that tumour necrosis factor (TNF) in the distal nephron contributes to nephrotoxicity.

The researchers previously identified that activation of AT1 receptors on T cells attenuated kidney damage in hypertension. Here, they examined whether the protective action of the T-cell AT1 receptor could also be observed in cisplatin-induced AKI, in which TNF is a known mediator of injury. The researchers genetically deleted AT1 receptors from $\mathrm{T}$ cells (TKO) or from the distal nephron (KKO) in wild-type mice, and then examined the susceptibility of these mice to cisplatin-induced AKI.

"The TKO mice had exaggerated AKI and TNF generation following cisplatin injection, whereas the KKO mice were partially protected from AKI and had blunted TNF generation," describes Crowley. "To confirm that TNF was important in protecting the KKO mice from injury we deleted TNF from the distal nephron and found that these mice were also protected from cisplatin-induced AKI."

Crowley proposes that these data are consistent with an important role for TNF in cisplatin-induced AKI, and suggests that blocking AT1 receptors in the kidneys but activating AT1 receptors on T cells might represent a future intervention to mitigate AKI once technologies emerge to enable tissue-specific therapies. "We now want to understand if the T-cell AT1 receptor functions in the pathogenesis of other forms of AKI, and whether AT1 receptors in specific tissues modulate the susceptibility to chronic kidney disease that occurs as a complication of AKI." 